\title{
THE ARRANGEMENT OF AXONS ACCORDING TO FIBER DIAMETER IN THE OPTIC TRACT OF THE CAT ${ }^{1}$
}

\author{
R. W. GUILLERY,*,2 E. H. POLLEY, 市 AND F. TORREALBA $\S$ \\ *Department of Pharmacological and Physiological Sciences, The University of Chicago, Chicago, Illinois 60637, \\ $\$$ Departments of Anatomy, Ophthalmology, and Neurosurgery, University of Illinois at the Medical Center, Chicago, Illinois \\ 60680, and \$Departamento de Fisiología y Biofísica, Iniversidad de Chile, Santiago, Chile
}

Received October 19, 1981; Revised January 4, 1982; Accepted January 4, 1982

\begin{abstract}
The topographic distribution of axons according to diameter classes in the optic tract of the cat has been studied by light and electron microscopic methods. The subdivision of the tract into a ventral and a dorsal sector, with the former containing the thickest fibers of the tract, has been confirmed. In addition, there is a ventrolateral marginal zone of the tract which contains many of the finest axons and there are gradients of fiber diameters definable for thick and intermediate axons. 'The gradients show the thinner fibers lying rostral to the thicker fibers. The major points demonstrable by fiber diameter measurements have been confirmed by counts of myelin lamellae. Comparison of fiber distributions in the optic tract with the distributions in the optic nerve on the one hand and with the geniculate terminations on the other shows that, in the region of the optic chiasm, retinofugal axons are sorted not only according to their crossed or uncrossed pathway but also according to their fiber diameter class and thus are presorted in terms of their final destinations.
\end{abstract}

Within the optic tract of the cat, there is a clear segregation of fiber diameter classes. This is readily seen in transverse or oblique sections prepared for light or electron microscopic study and has been described previously by Chang (1952, 1956), Bishop et al. (1953), and Bishop and Clare (1955) (see also Chang and Cheng, 1961; Bishop et al., 1969). The tract can be divided into two parts: a dorsomedial part within which there are no thick axons and a ventrolateral part within which thick fibers appear to dominate. During a study of the topographic organization of the fibers in the optic tract (Torrealba et al., 1981), we re-examined this segregation and noticed two features that have not been described previously. One is a gradient of fiber diameters that occurs within both of the subdivisions of the tract, the dorsomedial and the ventrolateral, and the other is a concentration of some of the finest fibers of the tract next to its ventral and lateral borders. Since both are of interest in understanding how different categories of retinofugal fibers reach their appropriate end stations, they are described briefly in the following.

\footnotetext{
I This work was supported by National Institutes of Health Grants NS-14283 and EY-02374. S. Price and J. Dobson were responsible for much of the histological processing and $S$. Price prepared the illustrations. M. Jinbo typed the manuscript. We are grateful for their help.

"To whom correspondence should be addressed at Department of Pharmacological and Physiological Sciences, The University of Chicago, 947 East 58th Street, Chicago, IL 60637.
}

\section{Materials and Methods}

Four normal adult cats were used. In addition, we used one cat that had had a small photocoagulation made in the retina above the area centralis and that survived 12 days after the photocoagulation. The number of degenerating fibers in the optic tract of this fifth cat was so small that the tract did not differ significantly from the normal. The animals were anesthetized with sodium pentobarbital and perfused with either $1 \%$ glutaraldehyde and $1 \%$ paraformaldehyde in $0.1 \mathrm{M}$ cacodylate buffer (at $\mathrm{pH} 7.4$ ) followed by $2 \%$ glutaraldehyde and $2 \%$ paraformaldehyde in the same buffer (one cat) or with $4 \%$ paraformaldehyde in a $0.1 \mathrm{M}$ phosphate buffer (at $\mathrm{pH}$ 7.4). After $24 \mathrm{hr}$ in the fixative, blocks containing the optic nerves and tracts were cut on a Vibratome at 100 to 400 $\mu \mathrm{m}$. These sections were rinsed in the buffer for 1 to $2 \mathrm{hr}$ and then were transferred to osmium tetroxide in the same buffer for 30 to $75 \mathrm{~min}$. The sections were dehydrated in ethanol and mounted flat in Durcupan (Ciba). Thin sections $(1 \mu \mathrm{m})$ were cut for light microscopy and stained with $1 \% \quad p$-phenylenediamine (Korneliussen, 1972). Thin sections for electron microscopy were stained with lead citrate.

Sections for electron microscopy were cut adjacent to the semithin sections so that the precise part of the tract from which electron micrographs were taken could be identified by reference to blood vessels, large myelinated axons, and other landmarks. Axon diameters were mea- 
sured from electron micrographs that were photographed at an initial magnification of $\times 1,500$ or $\times 2,000$ (magnification was checked by use of a diffraction grating replica) and which then were magnified $\times 2.9$. For each axon, we measured the maximum diameter and the diameter at right angles to this and took the mean of these two as the "mean diameter." Regions for these measurements were chosen on the light microscopic section, and within the same area of an adjacent electron micrograph, all axons were measured, starting at one edge of a rectangle and proceeding in orderly sequence to the other until an adequate sample (generally about 100 ) was obtained. The rare axon that was clearly not cut in a plane close to the perpendicular was omitted (less than $2 \%$ of the axons) as were axons cut through a node of Ranvier (see Hughes and Wässle, 1976). We made no correction for distortions produced by sectioning (Hughes and Wässle, 1976). A comparison between our semithin sections and our thin sections (drawn in relation to the squares of the copper grid) showed that the compression was less than $10 \%$. Since we were concerned with comparing the parts of the tract in a single section, we made all measurements for Figure 3 from the same section and all measurements for Figure 4 from another single section.

Counts of the number of myelin turns on axons were obtained directly from the image on the screen of the electron microscope (Philips 201) as viewed through a $(\times 9)$ binocular magnifier. For these counts, we used an initial magnification of $\times 30,000$ and again recorded every axon within a rectangle that had been photographed previously at a low magnification. Thus, the axons could be marked on the photograph as the lamellae were counted. Most of the axons seen in a section from a well preserved tract, that had been cut at an appropriate angle, showed one area where the lamellae could be counted. Fewer than $5 \%$ of the axons had lamellae that could not be counted, and since they included all but the finest axons, we ignored the slight error introduced by this.

\section{Results}

The clearest view of the fiber distribution in the optic tract is obtained from sections cut perpendicular to the fibers of the tract. Figure 1 is an outline obtained from such a section, which is oblique to the conventional anatomical planes and which cuts the tract where it crosses the medial border of the cerebral peduncle. In this figure, lines 1 to 4 , which were drawn on the basis of light microscopic inspection, show the approximate borders of different fiber groups. Thus, the coarsest fibers, which are $4 \mu \mathrm{m}$ or more in diameter (excluding myelin), almost all lie ventral and lateral to the line labeled 3. A

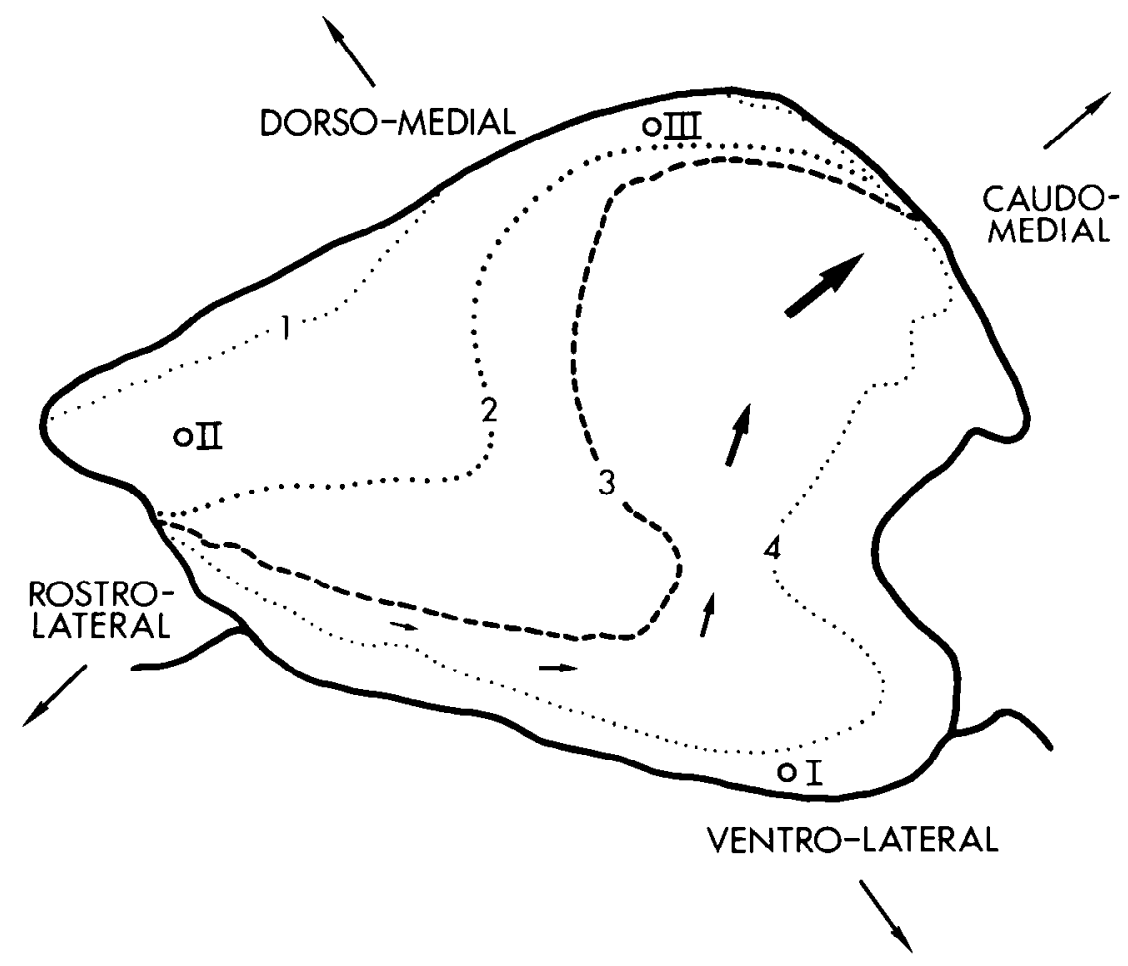

Figure 1. A schematic representation of a cross-section through the optic tract just lateral to the medial border of the cerebral peduncle. The plane of the section passes perpendicular to the fibers of the tract so that the directions indicated in the figure are somewhat oblique to the standard anatomical planes. The ventral parts of the section also lie lateral to the dorsal parts, and the rostral parts lie medial to the caudal parts. The part of the section ventral and lateral to line 3 is dominated by large axons greater than $4 \mu \mathrm{m}$ in diameter. However, there are significant numbers of fine $(1.5 \mu \mathrm{m}$ or less $)$ and intermediate axons scattered among the coarse ones. Ventral to line 4, the finest fibers are most prominent. Between lines 2 and 3 , there are fewer coarse fibers and the intermediate fibers dominate. Between lines 1 and 2, there are essentially no coarse fibers and most of the axons are of intermediate caliber. Dorsal to line 1, scattered fine fibers that probably belong to the supraoptic commissures dominate. $I$, $I I$, and $I I I$ show the approximate positions from which measurements and counts were taken, using another section from a different cat (sec Fig. 5 and text). The arrows indicate a gradient of fiber sizes (larger axons toward arrow heads) described in the text and illustrated in Figure 3. 
few coarse fibers lie between lines 2 and 3 , and none are seen dorsomedial to line 2. The photographs of Figure 2 and the histograms of Figure 3 also illustrate the differential distribution of the thickest fibers. However, while the thick fibers clearly dominate in the micrographs from the ventrolateral parts of the tract $(E, F, G$, and $H$ of Fig. 2), they form only a modest tail in the histograms for this part of the tract ( $I$ to $M$ of Fig. 3 ) and do not contribute more than $10 \%$ of the total fiber population in any of the histograms.

The majority of the axons near the outside of the tract, between the ventrolateral border and line 4 of Figure 1, are extremely fine, having diameters (excluding myelin) of less than $1.5 \mu \mathrm{m}$. Although these fibers clearly dominate in many of the histograms (e.g., $I$ to $M$ of Fig. 3), they are readily overlooked by mere inspection of the

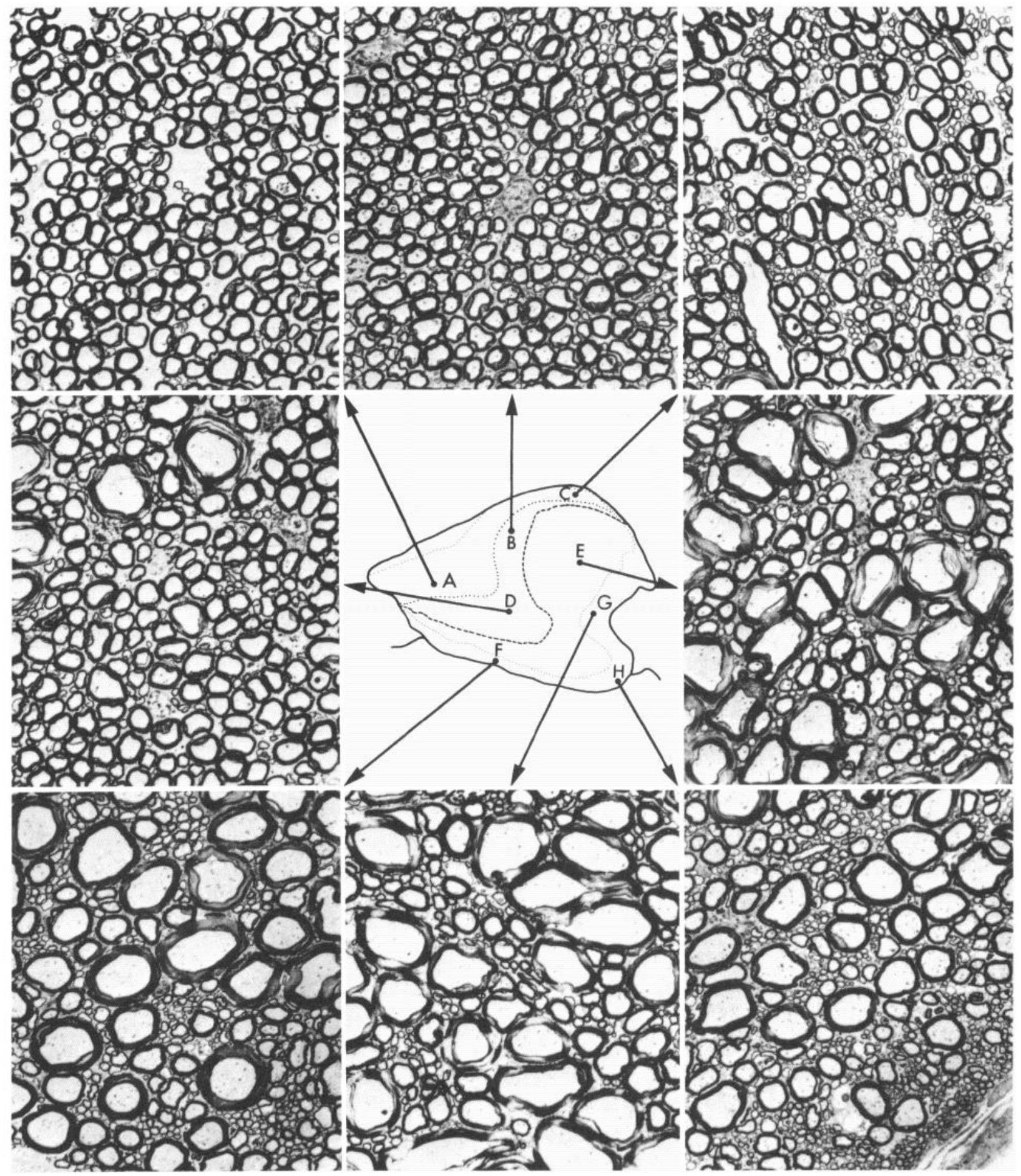

Figure 2. Low power electron micrographs from a section of the optic tract close to the level illustrated in Figure 1. The positions from which the micrographs were taken are shown on an outline of Figure 1. For further details, see text. Magnification $\times 1,200$. 


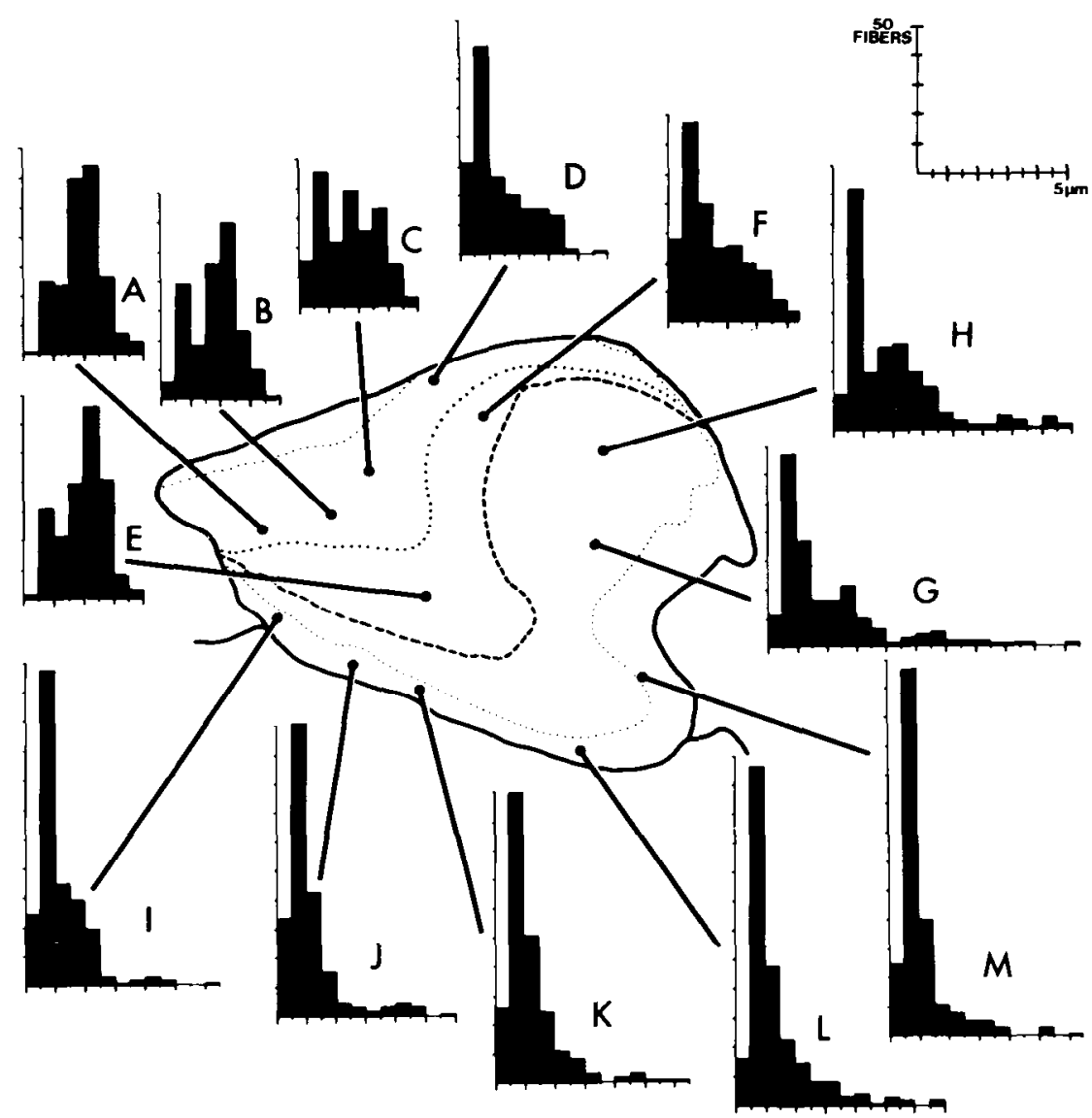

Figure 3. Distributions of fiber diameter classes within different parts of a cross-section of the optic tract close to the level of Figure 1 and superimposed upon an outline of that figure. For details, see text.

photographs of Figure 2. In fields progressively further from the ventrolateral border of the tract, one finds the proportion of these finest fibers decreasing markedly, and in the dorsomedial parts of the tract ( fields $A, B$, and $E$ of Fig. 3), there are relatively few fine fibers. However, some fine fibers can be found in all parts of the tract. They have a somewhat patchy distribution, especially in the midparts, between lines 2 and 4 of Figure 1, and there is some indication that small groups of fine axons are distributed preferentially adjacent to blood vessels.

Axons of medium caliber (approximately 2 to $4 \mu \mathrm{m}$ in diameter, excluding myelin) are distributed mainly between lines 1 and 3 of Figure 1, although there are some fibers of this diameter class in all parts of the tract. In a few areas, there appears to be an almost pure population of medium caliber axons (e.g., $A$ of Figs. 2 and 3), but more generally, the medium fibers are mingled with small fibers ( $C$ and $D$ of Fig. 2; $B, C$, and $E$ of Fig. 3) or with both small and large fibers ( $E$ and $G$ of Fig. 2; $G$ and $H$ of Fig. 3).

In the most dorsomedial parts of the tract, dorsal to line 1 of Figure 1, there is a heavy concentration of extremely fine axons. These probably belong to the supraoptic commissures. A significant proportion of these fine fibers is unmyelinated and since there are essentially no unmyelinated axons in the optic nerve (Hughes and
Wässle, 1976), the unmyelinated fibers in the tract are probably not retinofugal axons. We have not included any unmyelinated axons in the histograms. It is possible that the fine fiber peak of field $D$ (Fig. 3) represents a significant fine myelinated supraoptic component or even that supraoptic fibers extend further into the tract, but at present, we have no critical evidence on this point.

Inspection of a number of sections from different tracts confirms the conclusions presented above: the highest concentration of fine fibers occurs in a narrow outer rim of the tract, the coarsest fibers are largely confined to the ventrolateral half of the tract, and the dorsomedial half contains most of the medium size fibers as well as a significant number of scattered fine fibers.

Study of a number of tracts has shown that, in addition to the above, there is a modest gradient of fiber sizes that roughly follows the line of the arrows in Figure 1. This gradient is most evident for the coarsest axons and goes from finer to coarse in the direction of the arrows. The histograms of Figure 3 do not show this gradient very clearly because they are dominated by fine fibers, while the visible gradient involves the coarse fibers and, to a less obvious extent, the medium fibers. In order to demonstrate this gradient, we have measured the largest axons in the areas labeled $A$ to $E$ in Figure 4. In each area, axons having mean diameters (see "Materials and Methods") greater than $5 \mu \mathrm{m}$ (excluding myelin) were 

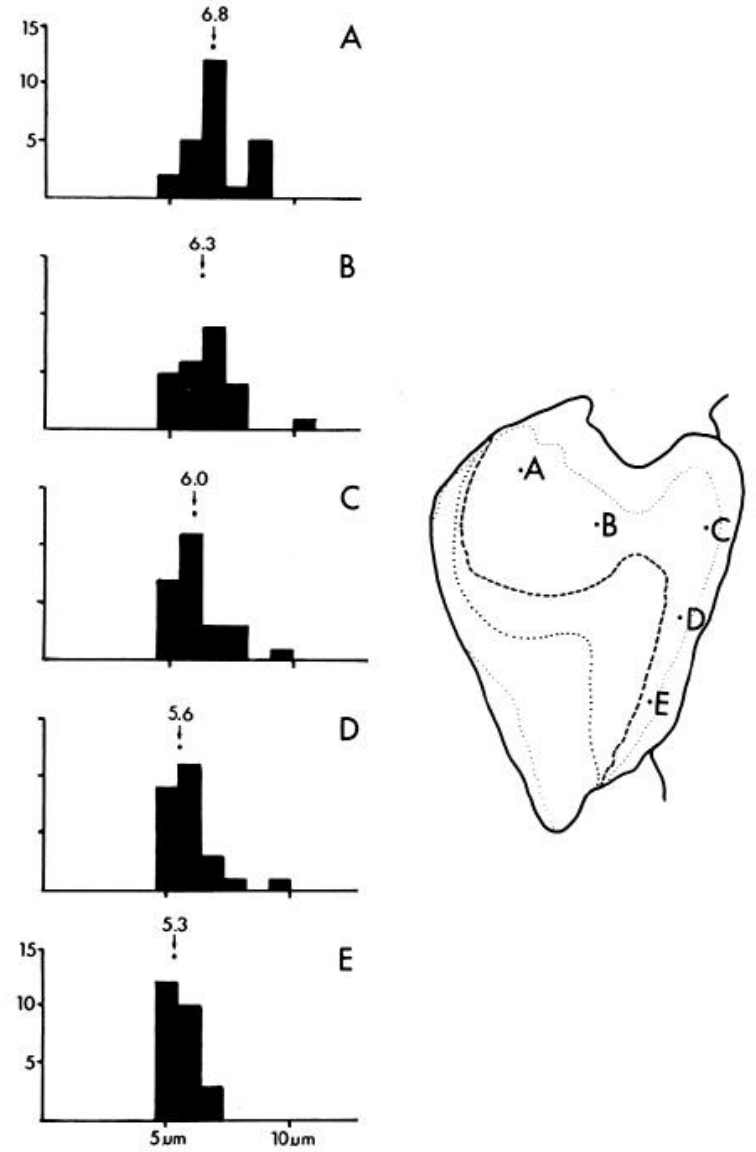

Figure 4. Distributions of measurements of the mean diameters of the 25 largest axons in areas $A$ to $E$, showing the gradient of fiber sizes indicated by the arrows in Figure 1 and described in the text.

measured in a semithin section under a $\times 100$ oil immersion objective. The axons to be measured were chosen by superimposing a set of concentric circles upon the microscopic field and measuring the axons sequentially from the center toward the outer circle until the sample included 25 fibers. The results, illustrated in Figure 4, show the gradient of fiber sizes for these coarsest axons clearly: the caliber of the thickest axons increases progressively as one moves from $E$ to $A$. A $t$ test shows that the mean measurement at $E$ is significantly different ( 0.05 probability level) from that at $A, B$, or $C$ and that, similarly, the mean at $A$ differs from that at $C, D$, or $E$.

Visual impressions suggest that there is a similar gradient for the medium fibers, although it appears to be less marked. Measurements taken from electron micrographs of regions corresponding closely to the areas marked $I I$ and $I I I$ in Figure 1, but from another block of the same tract, have confirmed this impression. In each area, the mean diameters of the 26 largest axons within a photographic montage were measured. For area II, the mean $( \pm \mathrm{SE})$ of these measurements was $3.67 \pm 0.017 \mu \mathrm{m}$ (excluding myelin), and for area III, it was $4.15 \pm 0.019$ $\mu \mathrm{m}$. The difference is significant at the 0.01 level of probability ( $t$ test) and suggests that there is a comparable gradient for the medium size fibers. For comparison, the mean obtained by the same methods from electron micrographs of area I was $5.64 \pm 0.018 \mu \mathrm{m}$. This is close to the value obtained for area $C$ of Figure 4.
Fiber diameter measurements give a fair estimate of size distributions in large populations, but since fibers in histological preparations of the retinofugal pathways have a tendency to be varicose and may be obliquely cut, it has been suggested that myelin thickness may offer a more reliable measure of axon size (Freeman, 1978). The number of myelin lamellae probably offers the most reliable measure, and in order to confirm some of the main conclusions of the axonal measurements, we have made counts of myelin lamellae in areas corresponding closely to the points marked $I, I I$, and $I I I$ in Figure 1 . As would be expected, there is a correlation between axon diameter and the number of myelin turns $(r=0.93$ for area $I ; r=0.75$ for area $I I ; r=0.86$ for area III). The results for area $I$ (Fig. 5I) show a predominant popula-
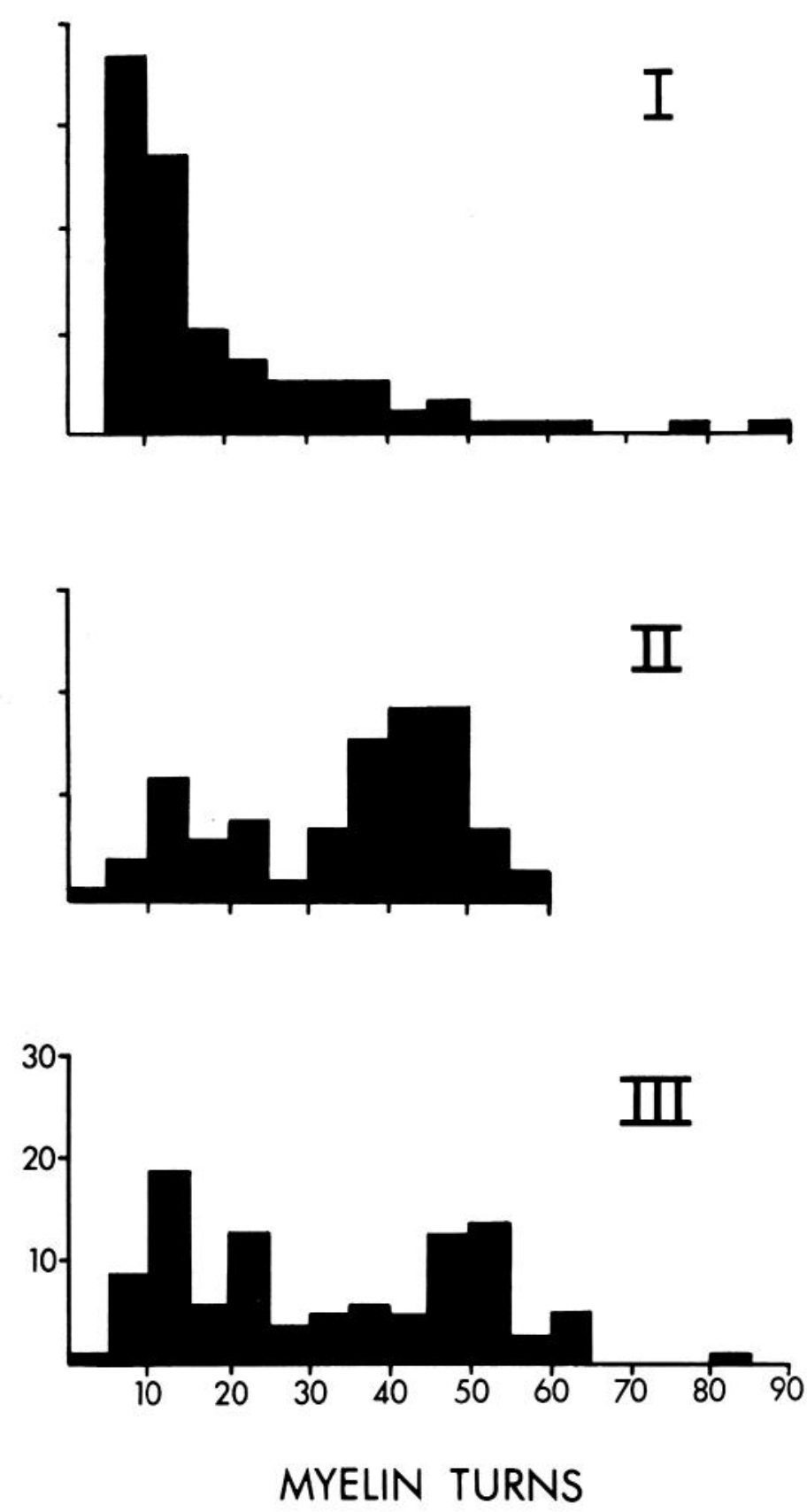

Figure 5. Distributions of counts of myelin lamellae for areas close to the regions marked $I, I I$, and $I I I$ in Figure 1 . 
tion of fine axons and a poorly marked tail of coarse fibers, essentially confirming the results shown in Figure $3(K, L$, and $M)$. The results from areas $I I$ and $I I I$ (Fig. 5) show fewer fine fibers and show the distinction between the fine and the medium fibers rather more clearly than did the diameter measurements of Figure 3. The slightly higher number of turns for the medium size fibers in area III than in area II confirms the gradient considered in the previous paragraph.

Counts of myelin turns of the 26 largest axons in each of the three areas (areas $I, I I$, and $I I I$ of Fig. 1) also confirm the results obtained by diameter measurements: they demonstrate clearly that the largest axons are to be found in area $I$ and that the largest axons in area III are somewhat larger than the largest axons in area II. Thus, the means and the standard errors for areas $I, I I$, and $I I I$ are $75.5 \pm 0.54,50.6 \pm 0.49$, and $56.3 \pm 0.45$ turns, respectively, and these means are significantly different from each other at a 0.05 probability level ( $t$ test).

Since most previous investigators who have measured retinofugal axons have reported their results in terms of total thickness including myelin, we measured 50 axons from one region of the optic tract and recorded axon diameter with and without myelin for each fiber. In view of the suggestion that, in retinofugal axons, myelin thickness represents a better measure of axon size than does axon diameter (Freeman, 1978), the correlation was surprisingly good $(r=0.99)$. The slope of the line relating the two measures was 1.39 , which is the correction that should be applied to our data in order to compare them with published measures of total axon thickness.

The correlations between axon diameter and number of myelin turns (p. 718), or between axon diameters with and without myelin, suggest that varicose irregularities are not a serious problem in optic tracts prepared by the methods that we have used. Since the main conclusions of our study concern differences between different parts of the optic tract, they are largely independent of possible artifacts introduced by varicose fibers. In any one region, the occurrence of varicosities can increase the variance of diameter measures but is not likely to shift the mean to a significant extent.

As one traces the optic tract from the crossing with the medial border of the cerebral peduncle toward the lateral geniculate nuclei, so one finds that the visually evident segregation of fiber diameter classes continues, with the coarsest fibers lying external to the medium fibers and a quite dense group of fine fibers lying near the outer border of the tract among the coarse fibers. At the level of the lateral geniculate nucleus, fibers begin to pass perpendicular to the main direction of the tract in order to enter the nucleus and the fiber segregation becomes somewhat less clear. However, as the tract envelops the dorsal lateral geniculate nucleus, it is still possible to recognize the basic pattern of the segregation, with the main population of medium fibers lying next to the nucleus and coarse and fine fibers lying nearer the outer, pial surface. Sagittal sections of the dorsal lateral geniculate nucleus stained with a nonsuppressive silver method occasionally show this trilaminar arrangement of the fibers particularly clearly in the tract as it wraps around the nucleus.

Tracing the tract toward the chiasm has proved less instructive. One can see bundles of thick fibers traversing the tract obliquely close to the chiasm, and it appears that these reflect some of the fiber rearrangement that occurs between the nerve and the tract (see Aebersold et al., 1981). Within the nerve itself, there is no obvious fiber segregation comparable to that seen in the tract. The course taken by the fine fibers near the chiasm has not been clearly revealed in our material.

\section{Discussion}

Our observations confirm previous descriptions of fiber diameter classes in the optic tract of the cat (Chang, 1952, 1956; Bishop et al., 1953; Bishop and Clare, 1955; Chang and Cheng, 1961; Aebersold et al., 1981) and extend them. In addition to the localization of the coarsest fibers within the ventrolateral parts of the tract recognized by others, we have found a marked concentration of finest fibers at the ventrolateral margins of the tract (Figs. 1 to 3 ) and have shown that, within the coarse and intermediate populations, there is a rostrocaudal gradient of axon sizes, with the coarsest fibers lying caudally. In view of these gradients, one cannot demarcate fiber categories accurately in terms of diameter only, but in general, the coarse component is made up of fibers having mean axon diameters (without myelin) greater than 4 to $5 \mu \mathrm{m}$, the intermediate component is 1.5 to 4 $\mu \mathrm{m}$, and the fine component is less than $1.5 \mu \mathrm{m}$ (see Fig. 3 ) in diameter. If myelin had been included in these measures, they would be: greater than 5.5 to $7 \mu \mathrm{m}$ for the coarse fibers, 2 to $5.5 \mu \mathrm{m}$ for the intermediate ones, and less than $2 \mu \mathrm{m}$ for the finest.

Chang $(1952,1956)$ described three distinct components in the optic tract of the cat with peaks at about 1 , 4, and $9 \mu \mathrm{m}$. P. O. Bishop et al. (1953) described two major components in the cat's optic tract: a dorsal fine one and a ventral coarse one. In addition, they found slight differences between the crossed and the uncrossed components. There is a hint in their published electrophysiological records (their Fig. 1, $d$ and $i$ ) of a third, yet finer fiber group in the most ventral parts of the tract in the crossed, but not the uncrossed, component. This would probably correspond to our marginal group of finest fibers, but it is merely a hint in their records and they did not discuss it.

G. H. Bishop and Clare (1955) described four components in the optic tract, which they distinguished in terms of conduction velocities and thresholds. Their correlated anatomical study showed only three classes (coarse, intermediate, and fine), because they were unable to distinguish the subdivision of the fine fibers. Their coarse fibers ( 8 to $12 \mu \mathrm{m}$ in diameter) lie in the lateral part of the tract and correspond to the largest fibers of our coarse component. Their intermediate fibers (having diameters of 4 to $8 \mu \mathrm{m}$ ) must correspond mainly to the smaller fibers of our coarse component, since the measures overlap and since, in their material, this group also lies in the lateral parts of the tract scattered among their "coarse" fibers. The possibility that our coarse component can be subdivided is clearly of interest but has not been tested in our material. The fibers that formed Bishop and Clare's fine component showed a peak in the $2-\mu \mathrm{m}$ range and were concentrated in the medial parts of the tract so that they correspond mainly 


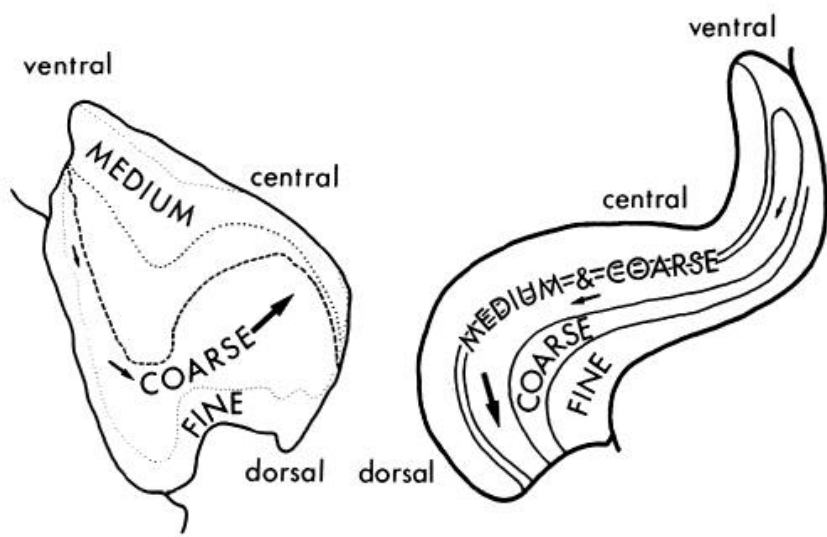

Figure 6. Schematic outlines of a section perpendicular to the optic tract (left) and of a parasagittal section through the dorsal lateral geniculate nucleus (right). The section of the tract has been turned through $90^{\circ}$ relative to Figure $1 .^{3}$ The distribution of fiber diameter classes (coarse, medium, and fine) is shown in the tract and their primary termination sites are shown in the nucleus. The representations of the retinal maps are indicated by dorsal, central, and ventral, while the arrows show gradients of fiber size (tract) or cell size, with larger elements grouped toward the arrow heads. Note that each geniculate layer and each fiber diameter group represents the whole retina, with the several maps being roughly in register in the nucleus and in the tract. The labels ventral, central, and dorsal are shown for only one edge of each structure, but the relevant retinal representations extend roughly perpendicular to this edge through the nucleus and tract.

to our intermediate group. Aebersold et al. (1981), using light microscopic methods, also described two major subdivisions of the optic tract, with thinner fibers lying dorsally and thicker fibers lying ventrally. These components thus also correspond to our intermediate and coarse fiber groups. It is to be noted that the relative position of the two groups changes as the tract curves around the diencephalon. The intermediate component maintains an internal position relative to the others all the way to the lateral geniculate nucleus (Bishop and Clare, 1955) and thus shifts from a dorsal to a medial position.

The relative position of the fiber components in the tract bears an interesting relation to their terminal distribution in the dorsal lateral geniculate nucleus, which is suggestive of a significant amount of "presorting" of the fibers before they reach their end stations. In Figure 6 , the outline of the tract used in Figures 1 to 4 has been turned through about $90^{\circ},{ }^{3}$ and the major directions of the retinotopic maps defined by Torrealba et al. (1981) have been added (they are shown as ventral, central, and dorsal retinal directions in the figure). The main concentrations of the coarse, medium, and fine axons are shown and the arrows indicate the gradient of increasing fiber diameters that we have found in the tract. On the right side of the figure is an outline of a parasagittal section through the dorsal lateral geniculate nucleus on which the major directions of the retinal maps (Sanderson,

\footnotetext{
${ }^{3}$ This corresponds roughly to the change in position undergone by the cat's dorsal lateral geniculate nucleus during postnatal development (Kalil, 1978), as it rotates in a parasagittal plane.
}

1971) and the terminal sites of the main fiber components are shown (Guillery, 1970, 1979; Cleland et al., 1975; Wilson et al., 1976; Rodieck, 1979). Also, the arrows on this figure indicate a gradient of cell sizes that characterizes the geniculate laminae (Guillery, 1970).

It can be seen that the pattern of fiber distributions in the tract matches the geniculate pattern closely so that a superposition of the two patterns can be produced rather simply by the rotation of the tract that occurs as it passes around the diencephalon (see Bishop and Clare, 1955). In general, within the retinogeniculate system, there is a good correlation between the size of geniculate relay cells and the size of the retinal afferents that innervate the cells (Rodieck, 1979; Stone et al., 1979; Sherman and Spear, 1982). Figure 6 indicates that this correlation may apply not merely to the major functional classes of the geniculocortical pathways (the "Y," "X," and "W" pathways) but that it also may apply to the gradients of fiber diameter that we have demonstrated in the tract. These gradients, and the related gradients of geniculate cell size, have not been studied in terms of possible functional correlates. It is recognized that, for each ganglion cell class, there are central:peripheral gradients of ganglion cell sizes related to receptive field sizes (see references cited above), but the gradient that we are considering here corresponds to the dorsoventral axis of the retina, with the largest axons and geniculate cells representing the dorsal retina and the smallest representing the ventral retina. The developmental or functional significance of this gradient has not been defined, nor is it known how it relates to the central:peripheral gradients, but it may be relevant that a major dorsoventral retinal difference is produced by the tapetum of the cat's retina, which must be related to some marked differences in the functional capacities of the two parts of the retina. However, one might expect that these are rather sharp dorsoventral differences, which may not correspond to the rather even gradient that we have found.

The geniculate component of the optic tract represents only one of several retinofugal fiber groups. We do not know how the fiber arrangements in the tract relate to terminal distributions within most of these other groups, but there is evidence that, in the superior colliculus, the finer "W" component is superficial to the coarser "Y" component (Hoffmann, 1973; McIlwain, 1973; McIlwain and Lufkin, 1976), which corresponds to the relationship between the finest and coarsest fibers in the tract. The intermediate component appears not to reach the colliculus.

The segregation of fiber classes so clearly evident in the optic tract is not seen in the optic nerve (Chang, 1956; van Crevel and Verhaart, 1963; Donovan, 1967; Hughes and Wässle, 1976; Stone and Campion, 1978). There is some indication that, behind the eye, the central and lateral parts of the nerve contain finer fibers, but this pattern changes further from the eye and is lost before the chiasm is reached. One can regard the fiber segregation in the nerve as representative of central:peripheral gradients, while that in the tract represents a separation of fiber classes anticipatory of their separate terminal distributions. The region of the chiasm must be regarded as a zone within which fibers are segregated not merely 
in terms of their pathway to the left or the right hemisphere but also in terms of their functional type.

\section{References}

Aebersold, H., O. D. Creutzfeldt, U. Kuhnt, and D. Sanides (1981) Representation of the visual field in the optic tract and optic chiasm of the cat. Exp. Brain Res. 42: 127-145.

Bishop, G. H., and M. H. Clare (1955) Organization and distribution of fibers in the optic tract of the cat. J. Comp. Neurol. 103: 269-304.

Bishop, G. H., M. H. Clare, and W. M. Landau (1969) Further analysis of fiber groups in the optic tract of the cat. Exp. Neurol. 24: 386-399.

Bishop, P. O., D. Jeremy, and J. W. Lance (1953) The optic nerve. Properties of a central tract. J. Physiol. (Lond.) 121: 415-432.

Chang, H. T. (1952) Functional organization of central visual pathways. Res. Publ. Assoc. Res. Nerv. Ment. Dis. 30: 430453.

Chang, H. T. (1956) Fiber groups in primary optic pathway of cat. J. Neurophysiol. 19: 224-231.

Chang, H. T., and T. H. Cheng (1961) An analysis of fibre constitution of optic tract of cat. Sci. Sin. 10: 538-556.

Cleland, B. G., R. Morstyn, H. G. Wagner, and W. R. Levick (1975) Long latency retinal input to lateral geniculate neurones in the cat. Brain Res. 91: 306-310.

Donovan, A. (1967) The nerve fibre composition of the cat optic nerve. J. Anat. 101: 1-11.

Freeman, B. (1978) Myelin sheath thickness and conduction latency groups in the cat optic nerve. J. Comp. Neurol. 181: 183-196.

Guillery, R. W. (1970) The laminar distribution of retinal fibers in the dorsal lateral geniculate nucleus of the cat: A new interpretation. J. Comp. Neurol. 138: 339-367.

Guillery, R. W. (1979) A speculative essay on geniculate lamination and its development. Prog. Brain Res. 51: 403-418.

Hoffmann, K. P. (1973) Conduction velocity in pathways from retina to superior colliculus in the cat: A correlation with receptive field properties. J. Neurophysiol. 36: 409-424.

Hughes, A., and H. Wässle (1976) The cat optic nerve: Fiber total count and diameter spectrum. J. Comp. Neurol. 169: 171-184.

Kalil, R. E. (1978) Development of the dorsal lateral geniculate nucleus in the cat. J. Comp. Neurol. 182: 265-292.

Korneliussen, H. (1972) Identification of muscle fiber types in semithin sections stained with paraphenylendiamine. Histochemie 32: 95-98.

Mcllwain, J. T. (1973) Topographic relationships in the projection from the striate cortex to the superior colliculus of the cat. J. Neurophysiol. 36: 690-707.

Mcllwain, J. T., and R. B. Lufkin (1976) Distribution of direct $\mathrm{Y}$-cell inputs to the cat's superior colliculus: Are there spatial gradients? Brain Res. 103: 133-138.

Rodieck, R. W. (1979) Visual pathways. Annu. Rev. Neurosci. 2: 193-225.

Sanderson, K. J. (1971) The projection of the visual field to the lateral geniculate and medial interlaminar nuclei in the cat. J. Comp. Neurol. 143: 101-118.

Sherman, S. M., and P. D. Spear (1982) Organization of the visual pathways in normal and visually deprived cats. Physiol. Rev., in press.

Stone, J., and J. E. Campion (1978) Estimate of the number of myelinated axons in the cat's optic nerve. J. Comp. Neurol. 180: 799-806.

Stone, J., B. Dreher, and A. Leventhal (1979) Hierarchical and parallel mechanisms in the organization of visual cortex. Brain Res. Rev. 1: 345-394.

Torrealba, F., R. W. Guillery, E. H. Polley, and C. A. Mason (1981) A demonstration of several independent, partially overlapping, retinotopic maps in the optic tract of the cat. Brain Res. 219: 428-432.

van Crevel, H., and W. J. C. Verhaart (1963) The rate of secondary degeneration in the central nervous system. II. The optic nerve of the cat. J. Anat. 97: 451-464.

Wilson, P. D., M. H. Rowe, and J. Stone (1976) Properties of relay cells in cat's lateral geniculate nucleus: A comparison of W-cells with X-and Y-cells. J. Neurophysiol. 39: 1193-1209. 